\title{
UNIQUE CONTINUATION ESTIMATES FOR THE LAPLACIAN AND THE HEAT EQUATION ON NON-COMPACT MANIFOLDS
}

\author{
LuC MiLleR
}

\begin{abstract}
This article concerns some quantitative versions of unique continuation known as observability inequalities. One of them is a lower bound on the spectral projectors of the Dirichlet Laplacian which generalizes the unique continuation of an eigenfunction from any open set $\Omega$. Another one is equivalent to the interior null-controllability in time $T$ of the heat equation with Dirichlet condition (the input function is a source in $(0, T) \times \Omega)$. On a compact Riemannian manifolds, these inequalities are known to hold for arbitrary $T$ and $\Omega$.

This article states and links these observability inequalities on a complete noncompact Riemannian manifold, and tackles the quite open problem of finding which $\Omega$ and $T$ ensure their validity. It proves that it is sufficient for $\Omega$ to be the exterior of a compact set (for arbitrary $T$ ), but also illustrates that this is not necessary. It provides a necessary condition saying that there is no sequence of balls going infinitely far "away" from $\Omega$ without "shrinking" in a generalized sense (depending on $T$ ) which also applies when the distance to $\Omega$ is bounded.
\end{abstract}

\section{Introduction.}

Let $M$ be a smooth connected complete $n$-dimensional Riemannian manifold with metric $g$ and boundary $\partial M$. When $\partial M \neq \emptyset, M$ denotes the interior and $\bar{M}=M \cup \partial M$. Let $\Delta$ denote the (negative) Dirichlet Laplacian on $L^{2}(M)$ with domain $D(\Delta)=H_{0}^{1}(M) \cap H^{2}(M)$. Let $\Omega$ be an open subset of $\bar{M}$ and let $T$ be a positive time.

To begin with, we formulate various quantitative versions of unique continuation on $M$ for the heat equation and the spectral projectors $\mathbf{1}_{\sqrt{-\Delta} \leqslant \mu}$, with references to their introduction and proof on a compact $M$. Besides their intrinsic significance, they have applications to the controllability of distributed systems and the estimation of its cost (e.g. heat conduction, plate vibrations). Then we state the main result which addresses the quite open problem of their validity in this non-compact setting by describing their logical relationship and by providing some sufficient and some necessary geometric conditions.

1.1. Controllability/observability for the heat equation. Consider the heat equation on $M$ with Dirichlet boundary condition and a source term located

Received April 29, 2004.

2000 Mathematics Subject Classification. 35B60, 35B37, 58J35, 58J05, 93B07. 
in $\Omega_{T}=(0, T) \times \Omega\left(\mathbf{1}_{\Omega_{T}}\right.$ denotes its characteristics function $)$ :

$$
\begin{aligned}
& \partial_{t} \phi-\Delta \phi=\mathbf{1}_{\Omega_{T}} f \text { on } \mathbb{R}_{t} \times M, \quad \phi=0 \text { on } \mathbb{R}_{t} \times \partial M, \\
& \phi(0)=\phi_{0} \in L^{2}(M), f \in L_{\text {loc }}^{2}\left(\mathbb{R} ; L^{2}(M)\right) .
\end{aligned}
$$

Definition 1. The heat equation on $M$ is said to be null-controllable in time $T$ from $\Omega$ if for all $\phi_{0} \in L^{2}(M)$ there is a control function $f \in L^{2}(\mathbb{R} \times M)$ such that the solution $\phi \in C^{0}\left([0, \infty), L^{2}(M)\right)$ of (1) satisfies $u=0$ at $t=T$.

By duality (cf. [DR77]), it is equivalent to the following quantitative version of unique continuation for solutions $u(t, x)=e^{t \Delta} u_{0}(x)$ of the heat equation without source term: $\partial_{t} u=\Delta u$ on $\mathbb{R}_{t} \times M, u=0$ on $\mathbb{R}_{t} \times \partial M$.

Definition 2. The heat equation on $M$ is said to be final-observable in time $T$ from $\Omega$ if there is a positive constant $C_{T, \Omega}$ such that:

$$
\forall u_{0} \in L^{2}(M), \quad\left\|e^{T \Delta} u_{0}\right\|_{L^{2}(M)} \leqslant C_{T, \Omega}\left\|e^{t \Delta} u_{0}\right\|_{L^{2}\left(\Omega_{T}\right)} .
$$

The best constant $C_{T, \Omega}$ in (2) can be considered as the controllability cost since it is also the best constant such that $\|f\|_{L^{2}\left(\Omega_{T}\right)} \leqslant C_{T, \Omega}\left\|u_{0}\right\|_{L^{2}(M)}$, for all intial condition $u_{0}$ and control $f$ solving the controllability problem in def.1.

When $M$ is compact, Lebeau and Robbiano proved in [LR95] that these controllability/observability properties hold (for arbitrary $T$ and $\Omega$ ). In [MZ03], it is mentioned that their approach does not apply to unbounded Euclidean domains (indeed Weyl's asymptotics for eigenvalues and Russell's construction of biorthogonal functions are used in [LR95]) and that the only result available on these specific properties is that they hold when $M$ is a domain of the Euclidean space with the flat metric and the exterior of $\Omega$ is bounded (cf. [CDMZ01]). To this open problem, this article contributes the adaptation of the LebeauRobbiano approach to a non-compact $M$, the extension of the sufficient condition in [CDMZ01] and the necessary condition in [Mil04b] to a non-Euclidean $M$, and a finer sufficient condition for a homogeneous $M$ which applies even if the exterior of $\Omega$ is not compact.

1.2. Observability at low-frequencies for the heat and the Laplacian. In [LR95], Lebeau and Robbiano take advantage of the fast damping of high frequencies to reduce def.2 to the following estimate on the cost of fast nullcontrollability at low-frequencies.

Definition 3. Fast observability at low-frequencies for the heat equation on $M$ from $\Omega$ holds if there are positive constants $D_{1}$ and $D_{2}$ such that for all $\tau \in(0,1]$ and $\mu \geqslant 1$ :

$$
\forall u_{0} \in \mathbf{1}_{\sqrt{-\Delta} \leqslant \mu} L^{2}(M), \quad\left\|e^{\tau \Delta} u_{0}\right\|_{L^{2}(M)} \leqslant \frac{D_{2}}{\sqrt{\tau}} e^{D_{1} \mu}\left\|e^{t \Delta} u_{0}\right\|_{L^{2}\left(\Omega_{\tau}\right)} .
$$

When $M$ is compact, this improvement of prop.1 in [LR95] (allowing $\gamma$ to take the endpoint value $\gamma=1$ ) is a corollary of prop.2 in [LZ98]. In [LR95], the parabolic problem was reduced to a space-time elliptic one following a classical idea in the uniqueness for parabolic equations (cf. [Lin90]). The improvement 
in [LZ98] was obtained by first reducing def.3 to the following lower bound for $\mathbf{1}_{\Omega} \mathbf{1}_{\sqrt{-\Delta} \leqslant \mu}$, i.e. the restriction of the spectral projectors to $\Omega$.

Definition 4. Observability at low-frequencies for the Laplacian on $M$ from $\Omega$ holds if there are positive constants $C_{1}$ and $C_{2}$ such that for all $\mu>0$ :

$$
\forall v \in \mathbf{1}_{\sqrt{-\Delta} \leqslant \mu} L^{2}(M), \quad\|v\|_{L^{2}(M)} \leqslant C_{2} e^{C_{1} \mu}\|v\|_{L^{2}(\Omega)} .
$$

When $M$ is compact, this is an inequality on sums of eigenfunctions proved as th.3 in [LZ98] and th.14.6 in [JL99]. In particular, when $v$ is an eigenfunction of $\Delta$, this is a quantitative version of elliptic unique continuation. This spectral property should have wide applications besides the study of nodal sets (cf. [JL99]) and the control of vibrations (cf. [Mil04a]).

1.3. Carleman inequalities and interpolation. In [LZ98] and [JL99], this observability at low-frequencies for the Laplacian is reduced to the following interpolation inequality for the space-time Laplacian $P=\Delta+\partial_{t}^{2}$ on $M_{T}=$ $(0, T) \times M$ with Dirichlet condition on the "parabolic" boundary $\Gamma=\left(\mathbb{R}_{t} \times\right.$ $\partial M) \times(\{0\} \times \bar{M})$ and with observation of the Neumann condition on $\{0\} \times \Omega$.

Definition 5. The Laplacian $P$ satisfies the interpolation inequalities in time $T$ from $\Omega$ if $\forall \alpha \in(0,1 / 2), \exists \delta \in(0,1), \exists C>0, \forall \psi \in H^{2}\left(M_{T}\right)$ s.t. $\psi=0$ on $\Gamma$,

$$
\|\psi\|_{H^{1}((\alpha T,(1-\alpha) T) \times M)} \leqslant C\|\psi\|_{H^{1}\left(M_{T}\right)}^{\delta}\left(\|P \psi\|_{L^{2}\left(M_{T}\right)}+\left\|\partial_{t} \psi_{\rceil t=0}\right\|_{L^{2}(\Omega)}\right)^{1-\delta} .
$$

When $M$ is compact, this is lemma.A in [LZ98] and lemma.14.5 in [JL99] (it is also related to lemma.4.3 in [Lin91]). Following classical ideas in unique continuation for elliptic equations, the interpolation inequalities are based on Carleman-type estimates. The needed type, namely Carleman estimate for boundary problems, were introduced by Robbiano in [Rob95]. The specific Carleman inequalities used in [LZ98] and [JL99] are proved in prop.1 and prop.2 of [LR95].

1.4. The theorems. Before stating the main result, we introduce a geometric condition. The fundamental tone $\lambda^{*}(B)$ of an open subset $B$ of $\bar{M}$ is:

$$
\lambda^{*}(B)=\inf _{\varphi \in C_{0}^{\infty}(B) \backslash\{0\}} \frac{\|\nabla \varphi\|_{L^{2}(M)}^{2}}{\|\varphi\|_{L^{2}(M)}^{2}} .
$$

When $B$ is smooth and compact (e.g. $B$ is a ball with radius lower than the injectivity radius), then $\lambda^{*}(B)$ is just the first eigenvalue of the Dirichlet Laplacian on $B$ in the classical sense. In particular, the Euclidean ball $B_{r}$ with radius $r$ satisfies $\lambda^{*}\left(B_{r}\right)=\lambda^{*}\left(B_{1}\right) / r^{2}$, and the balls $B(x, r)$ of center $x \in M$ and radiusxs $r$ satisfy $\lambda^{*}(B(x, r)) \sim \lambda^{*}\left(B_{1}\right) / r^{2}$ as $r \rightarrow 0$. (Cf. e.g. sect.3.9 in [Cha93].)

Definition 6. The sequence of $\left(B_{k}\right)_{k \in \mathbb{N}}$ of open subsets of $\bar{M}$ goes "away" from $\Omega$ without "shrinking" with a factor $T$ if $\overline{\operatorname{dist}}_{T}\left(B_{k}, \Omega\right)^{2}-4 T^{2} \lambda^{*}\left(B_{k}\right) \rightarrow+\infty$ as 
$k \rightarrow+\infty$, where $\overline{\operatorname{dist}}_{T}$ is the following averaged distance with Gaussian weight of variance $T$ :

$\overline{\operatorname{dist}}_{T}(B, \Omega)^{2}=-2 T \log \int_{\Omega \backslash B} \exp \left(-\frac{\operatorname{dist}(x, B)^{2}}{2 T}\right) d x \geqslant \operatorname{dist}(B, \Omega)^{2}-2 T \operatorname{vol}(\Omega)$.

In [Mil04b], a necessary condition similar to def.6 was proved in the Euclidean case (using pointwise rather than $L^{2}$ Gaussian upper bounds) and illustrated by elementary examples. In particular, it applies even if the distance to $\Omega$ is bounded.

REMARK 1. Assuming that $\lambda_{\kappa, r}^{*}$ is the fundamental tone of the sphere of radius $r$ in the space form of constant sectional curvature $\kappa \in \mathbb{R}$ and that the Ricci curvatures of $M$ are all greater than or equal to $(n-1) \kappa$, we have $\lambda^{*}(B) \leqslant \lambda_{\kappa, r}^{*}$ for every ball $B$ of radius $r$ (cf. [Che75] or th.3.24 of [Cha93]). If the volume of $\Omega$ is finite and the radii of the balls $B_{k}$ are all greater than a positive constant, then def.6 just says that the geodesic distance from $B_{k}$ to $\Omega$ tends to infinity.

Theorem 1. Each of the following statements implies the next one:

i) $\Omega$ is the non-empty exterior of a compact set $K$ such that $K \cap \bar{\Omega} \cap \partial M=\emptyset$.

ii) $\partial_{t}^{2}+\Delta$ satisfies the interpolation inequalities in time $T$ from $\Omega$ (def.5).

iii) Observability at low-frequencies for the Laplacian on $M$ from $\Omega$ holds (def.4).

iv) Fast observability at low-frequencies for the heat on $M$ from $\Omega$ holds (def.3).

v) The heat equation on $M$ is final-observable in time $T$ from $\Omega$ (def.2).

vi) There is no sequence $\left(B_{k}\right)_{k \in \mathbb{N}}$ of open subsets of $\bar{M}$ going "away" from $\Omega$ without "shrinking" with a factor $T$ (def.6).

To obtain a sufficient condition for these observability inequalities that would be further from the case of a compact $M$ than i) in th.1, one is lead to make some assumptions on the geometry of $M$. For instance, if $M$ is a homogeneous manifold, a uniform version of def.5 holds: for any given $R>r>0$ and $\alpha \in$ $(0,1 / 2), \exists \delta \in(0,1), \exists C>0, \forall x \in \bar{M}, \forall \psi \in H^{2}\left(M_{T}\right)$ s.t. $\psi=0$ on $\Gamma$,

$$
\|\psi\|_{H^{1}\left((\alpha T,(1-\alpha) T) \times B_{R}\right)} \leqslant C\|\psi\|_{H^{1}\left(M_{T}\right)}^{\delta}\left(\|P \psi\|_{L^{2}\left(M_{T}\right)}+\left\|\partial_{t} \psi_{\rceil t=0}\right\|_{L^{2}\left(B_{r}\right)}\right)^{1-\delta},
$$

where $B_{r}$ and $B_{R}$ are the balls with center $x$ and respective radii $r$ and $R$. Indeed, for fixed $x$ this interpolation inequality results from lem.2.3, and $\delta$ and $C$ do not depend on $x$ since $M$ is homogeneous. The next theorem relaxes the assumption that the uncontrolled region $\bar{M} \backslash \Omega$ be compact.

Theorem 2. If the Ricci curvature of $M$ is bounded below and the uniform interpolation inequalities (6) hold (e.g. if $M$ is homogeneous), then th.1 holds with i) replaced by:

i') There are positive constants $r$ and $d$ such that any point in $\bar{M}$ is at a distance smaller than $d$ of some ball of radius $r$ included in $\Omega$. 
It would be interesting to know which boundedness assumptions on the geometry of $M$ ensure (6).

\section{Sufficient condition: $i) \Rightarrow i i)$}

The key to the proof of $i) \Rightarrow i i$ ) is the following global Carleman inequality with boundary term, similar to the local one in prop.1 of [LR95]. Taking advantage of the structure of $P$, it provides unique continuation with respect to $t$ only, but in terms of integral quantitities on the whole manifold $M$.

Let $\mathcal{F}$ be the space of functions $f \in C^{\infty}(\mathbb{R} \times \bar{M})$ such that $\operatorname{supp}(f) \subset$ $(-\infty, T) \times \bar{M}$ and $f_{\rceil[0, T] \times \partial M}=0$. Let $\mathcal{F}_{0}$ be the space of functions $f \in \mathcal{F}$ such that $f_{\rceil t=0}=0$.

Proposition 2.1. Let $\varphi: \mathbb{R} \rightarrow \mathbb{R}$ be a smooth function of time such that $\dot{\varphi}(t) \neq$ 0 and $\ddot{\varphi}(t)>0$ for all $t \in[0, T]$. There are positive constants $h_{0}$ and $C$ such that for all $h \in\left(0, h_{0}\right)$ and $g \in \mathcal{F}_{0}$ :

$$
\int_{M_{T}}\left|h^{2} P g\right|^{2} e^{\frac{2 \varphi}{h}}+h \int_{\{0\} \times M}\left|h \partial_{t} g\right|^{2} e^{\frac{2 \varphi}{h}} \geqslant C h \int_{M_{T}}\left(|g|^{2}+\left|h \partial_{t} g\right|^{2}+|h \nabla g|^{2}\right) e^{\frac{2 \varphi}{h}} .
$$

Proof. For each $f \in \mathcal{F}$, let $f_{0}=f_{\rceil t=0}$. Let $(f, g)=\int_{M_{T}} f \bar{g},\|f\|^{2}=(f, f)$, $(f, g)_{0}=\int_{M} f_{0} \bar{g}_{0},\|f\|_{0}^{2}=(f, f)_{0}$. We also introduce standard semiclassical notations where $h \in(0,1)$ is a (small) parameter: $D_{t}=\frac{h}{i} \partial_{t}, \nabla_{x}=\frac{h}{i} \nabla, \Delta_{x}=$ $-h^{2} \Delta,\|f\|_{1}^{2}=\|f\|^{2}+\left\|D_{t} f\right\|^{2}+\left\|\Delta_{x} f\right\|^{2}$. With $f=e^{\varphi / h} g$ and $P_{\varphi}=h^{2} e^{\varphi / h} \circ$ $P \circ e^{-\varphi / h}$, the Carleman inequality (7) writes:

$$
\left\|P_{\varphi}\right\|^{2}+h\left\|D_{t} f\right\|_{0}^{2} \geqslant C h\|f\|_{1}^{2} .
$$

We denote by $\varphi_{k}$ the derivative of order $k$ of $\varphi$, and let $\underline{\varphi}_{k}=\inf _{[0, T]}\left|\varphi_{k}\right|$, $\bar{\varphi}_{k}=\sup _{[0, T]}\left|\varphi_{k}\right|$. As usual, we introduce the hermitian and anti-hermitian parts of $P_{\varphi}$ :

$$
\begin{aligned}
& A=\frac{P_{\varphi}+P_{\varphi}^{*}}{2}=D_{t}^{2}+\Delta_{x}-\varphi_{1}^{2} \text { and } \\
& \qquad B=\frac{P_{\varphi}-P_{\varphi}^{*}}{2 i}=D_{t} \varphi_{1}+\varphi_{1} D_{t}=2 \varphi_{1} D_{t}-h \varphi_{2},
\end{aligned}
$$

Since $P_{\varphi}=A+i B$, we have $\left\|P_{\varphi} f\right\|^{2}=\|A f\|^{2}+\|B f\|^{2}+i[(B f, A f)-(A f, B f)]$. Since by integration by parts $(g, A f)=(A g, f)-i h\left(g, D_{t} f\right)_{0}$ and $(g, B f)=$ $(B g, f)$ for all $f \in \mathcal{F}_{0}$ and $g \in \mathcal{F}$, we deduce that:

$$
\left\|P_{\varphi} f\right\|^{2}=\|A f\|^{2}+\|B f\|^{2}+i([A, B] f, f)+2 h\left(\varphi_{1} D_{t} f, D_{t} f\right)_{0} .
$$

The computation of the commutator of $A$ and $B$, after the simplifications:

$$
\begin{aligned}
& {[A, B]=\left[D_{t}^{2}-\varphi_{1}^{2}, D_{t} \varphi_{1}+\varphi_{1} D_{t}\right]=} \\
& D_{t}\left[D_{t}^{2}, \varphi_{1}\right]+\left[D_{t}^{2}, \varphi_{1}\right] D_{t}+\varphi_{1}\left[D_{t}, \varphi_{1}^{2}\right]+\left[D_{t}, \varphi_{1}^{2}\right] \varphi_{1},
\end{aligned}
$$


yields $i[A, B]=4 h \varphi_{2}\left(D_{t}^{2}+\varphi_{1}^{2}\right)-h^{3} \varphi_{4}=4 h \varphi_{2}\left(A-\Delta_{x}+\varphi_{1}^{2}\right)-h^{3} \varphi_{4}$. Therefore,

$$
i([A, B] f, f)=4 h\left(\left(A f, \varphi_{2} f\right)+\left\|\sqrt{\varphi_{2}} \nabla_{x} f\right\|^{2}+2\left\|\varphi_{1} f\right\|^{2}\right)-h^{3}\left(\varphi_{4} f, f\right) .
$$

Since $\left|\left(A f, 4 h \varphi_{2} f\right)\right| \leqslant\|A f\|^{2} / 4+(4 h)^{2}\left\|\varphi_{2} f\right\|^{2}$, we deduce:

$$
i([A, B] f, f) \geqslant h\left(4 \underline{\varphi}_{2}\left\|\nabla_{x} f\right\|^{2}+\left(8 \underline{\varphi}_{1}^{2}-16 h \bar{\varphi}_{2}^{2}\right)\|f\|^{2}\right)-\|A f\|^{2} / 4 .
$$

Since $2\left|\left(\varphi_{1} D_{t} f, 2 h \varphi_{2} f\right)\right| \leqslant\left\|\varphi_{1} D_{t} f\right\|^{2}+(2 h)^{2}\left\|\varphi_{2} f\right\|^{2}$, we have:

$$
\begin{aligned}
& \|B f\|^{2}=\left\|2 \varphi_{1} D_{t} f\right\|^{2}+\left\|h \varphi_{2} f\right\|^{2}-2 \operatorname{Re}\left(\varphi_{1} D_{t} f, 2 h \varphi_{2} f\right) \geqslant \\
& \qquad \underline{\varphi}_{1}^{2}\left\|D_{t} f\right\|^{2}-5 h^{2} \bar{\varphi}_{2}^{2}\|f\|^{2} .
\end{aligned}
$$

Plugging this inequality and (10) in (9) yields:

$$
\begin{gathered}
\left.\left\|P_{\varphi} f\right\|^{2} \geqslant(3 / 4)\right)\|A f\|^{2}+h C_{h}\|f\|_{1}^{2}+2 h\left(\varphi_{1} D_{t} f, D_{t} f\right)_{0} \geqslant \\
h C_{h}\|f\|_{1}^{2}-2 h \bar{\varphi}_{1}\left\|D_{t} f\right\|_{0}^{2},
\end{gathered}
$$

with $C_{h}=\min \left\{8 \underline{\varphi}_{1}^{2}-21 h \bar{\varphi}_{2}^{2}, 4 \underline{\varphi}_{2}, \underline{\varphi}_{1}^{2}\right\}$. Taking $h_{0}>0$ small enough, so that $C_{h_{0}}>0$ yields (8) for all $h \in\left(0, h_{0}\right)$, which completes the proof of prop.2.1.

This global Carleman inequality implies (as in lemma.3 of [LR95]) the following global interpolation inequality whith the notations of def.5:

Lemma 2.2. Let $U$ be an open subset of $\bar{M}$ such that $\bar{U} \subset \Omega . \forall \tau \in(0, T)$, $\exists \delta \in(0,1), \exists C>0, \forall \psi \in H^{2}\left(M_{T}\right)$ s.t. $\psi=0$ on $\Gamma$,

$$
\begin{aligned}
& \|\psi\|_{H^{1}\left(U_{\tau}\right)} \leqslant \\
& \quad C\|\psi\|_{H^{1}\left(\Omega_{T} \backslash \Omega_{\tau}\right)}^{\delta}\left(\|P \psi\|_{L^{2}\left(M_{T}\right)}+\|\psi\|_{H^{1}\left((\Omega \backslash U)_{\tau}\right)}+\left\|\partial_{t} \psi_{\rceil t=0}\right\|_{L^{2}(\Omega)}\right)^{1-\delta} .
\end{aligned}
$$

Proof. Let $\tau^{\prime} \in(\tau, T)$ and let $\chi_{1}: \mathbb{R} \rightarrow[0,1]$ be a smooth function such that $\chi_{1}(t)=1$ for $t \leqslant \tau$ and $\chi_{1}(t)=0$ for $t \geqslant \tau^{\prime}$. Let $\chi_{2}: \bar{M} \rightarrow[0,1]$ be smooth function such that $\operatorname{supp}\left(\chi_{2}\right) \subset \Omega$ and $\chi_{2}=1$ on $U$. Applying prop.2.1 with $\phi(t)=\exp (-t)$ and $g=\chi \psi$ where $\chi=\chi_{1} \chi_{2}$, using $h<1$ and dividing by $h^{3}$, yields positive constants $C_{0}$ and $h_{0}<1$ such that for any $\psi$ as in lem.2.2 and all $h \in\left(0, h_{0}\right)$ :

$$
h \int_{M_{T}}|(P \psi,[P, \chi] \psi)|^{2} e^{\frac{2 \varphi}{h}}+\int_{\{0\} \times \Omega}\left|\partial_{t} \psi\right|^{2} e^{\frac{2 \varphi}{h}} \geqslant C_{0} \int_{U_{T}}\left|\left(\psi, \partial_{t} \psi, \nabla \psi\right)\right|^{2} e^{\frac{2 \varphi}{h}} .
$$

Since $\varphi$ is decreasing, $\phi(0)=1, \operatorname{supp}([P, \chi]) \subset(\Omega \backslash U)_{\tau} \cup\left(\Omega_{\tau^{\prime}} \backslash \Omega_{\tau}\right)$ and $[P, \chi]$ is a differential operator of order one, there is a $C_{1}>0$ depending on $\chi$ such that:

$$
\begin{aligned}
& h e\|P \psi\|_{L^{2}\left(M_{T}\right)}^{2}+h C_{1} e\|\psi\|_{H^{1}\left((\Omega \backslash U)_{\tau}\right)}^{2} \\
& +h C_{1} e^{\frac{2 \varphi\left(\tau^{\prime}\right)}{h}}\|\psi\|_{H^{1}\left(\Omega_{\tau^{\prime}} \backslash \Omega_{\tau}\right)}^{2}+e\left\|\partial_{t} \psi \psi_{\rceil=0}\right\|_{L^{2}(\Omega)}^{2} \\
& \qquad \geqslant C_{0} e^{\frac{2 \varphi(\tau)}{h}}\|\psi\|_{H^{1}\left(U_{\tau}\right)}^{2} \text { for all } h \in\left(0, h_{0}\right)
\end{aligned}
$$


Therefore, there are constants $h_{1} \in\left(0, h_{0}\right), D_{0}>0, D_{1}>1-\varphi(\tau)>0$, and $D_{2}=\varphi(\tau)-\phi\left(\tau^{\prime}\right)>0$ such that:

$$
\begin{aligned}
& e^{\frac{D_{1}}{h}}\left(\|P \psi\|_{L^{2}\left(M_{T}\right)}+\|\psi\|_{H^{1}\left((\Omega \backslash U)_{\tau}\right)}+\left\|\partial_{t} \psi_{\urcorner t=0}\right\|_{L^{2}(\Omega)}\right)+e^{-\frac{D_{2}}{h}}\|\psi\|_{H^{1}\left(\Omega_{\tau^{\prime}} \backslash \Omega_{\tau}\right)} \\
& \geqslant D_{0}\|\psi\|_{H^{1}\left(U_{\tau}\right)}, \quad \text { for all } h \in\left(0, h_{1}\right) .
\end{aligned}
$$

Optimizing with respect to $h$ as in [Rob95] completes the proof of lem.2.2.

Gluing together local interpolation inequalities (as in sect.3.B of [LR95]) on a fixed compact set intersecting $\Omega$ yields the following global interpolation inequality. (The first term of its left-hand-side corresponds to (2) in sect.3 of [LR95] with $t$ and $x$ interverted, whereas the second term corresponds to (1).)

Lemma 2.3. For all compact subsets $K^{\prime}$ and $K^{\prime \prime}$ such that $K^{\prime} \subset M$, for all segment $S \subset(0, T), \forall \tau \in(0, T), \exists \delta \in(0,1), \exists C>0, \forall \psi \in H^{2}\left(M_{T}\right)$ s.t. $\psi=0$ on $\Gamma$,

$\|\psi\|_{H^{1}\left(K_{\tau}^{\prime}\right)}+\|\psi\|_{H^{1}\left(S \times K^{\prime \prime}\right)} \leqslant C\|\psi\|_{H^{1}\left(M_{T}\right)}^{\delta}\left(\|P \psi\|_{L^{2}\left(M_{T}\right)}+\left\|\partial_{t} \psi_{\rceil t=0}\right\|_{L^{2}(\Omega)}\right)^{1-\delta}$.

Assume $i$ ), i.e. $\bar{M} \backslash K=\Omega \neq \emptyset$ for some compact subset $K$ of $\bar{M}$ such that $K \cap \bar{\Omega} \subset M$. Let $V$ be an open subset of $\bar{M}$ containing $K \cap \bar{\Omega}$ such that $\bar{V}$ is compact and $\bar{V} \subset M$. Applying lem.2.2 to $U=\Omega \backslash \bar{V}$ using $\Omega_{T} \backslash \Omega_{\tau} \subset M_{T}$ and $\Omega \backslash U \subset \bar{V}$, and applying lem.2.3 to $K^{\prime}=\bar{V}, K^{\prime \prime}=K, S=[\alpha T,(1-\alpha) T]$ and $\tau=(1-\alpha) T$ yield: $\exists \delta^{\prime} \in(0,1), \exists C^{\prime}>0, \forall \psi \in H^{2}\left(M_{T}\right)$ s.t. $\psi=0$ on $\Gamma$,

$$
\begin{aligned}
\|\psi\|_{H^{1}\left(\Omega_{(1-\alpha) T}\right)} & \leqslant C^{\prime} A^{\delta^{\prime}}\left(B+\|\psi\|_{H^{1}\left(V_{(1-\alpha) T}\right)}\right)^{1-\delta^{\prime}}, \\
\|\psi\|_{H^{1}\left(V_{(1-\alpha) T}\right)} & \leqslant C^{\prime} A^{\delta^{\prime}} B^{1-\delta^{\prime}} \\
\|\psi\|_{H^{1}((\alpha T,(1-\alpha) T) \times K)} & \leqslant C^{\prime} A^{\delta^{\prime}} B^{1-\delta^{\prime}},
\end{aligned}
$$

with $A=\|\psi\|_{H^{1}\left(M_{T}\right)}$ and $B=\|P \psi\|_{L^{2}\left(M_{T}\right)}+\left\|\partial_{t} \psi_{\urcorner t=0}\right\|_{L^{2}(\Omega)}$. Plugging (12) into (11) as in lem.4 of [LR95] yields the estimate $\|\psi\|_{H^{1}\left(\Omega_{(1-\alpha) T}\right)} \leqslant C^{\prime \prime} A^{\delta^{\prime \prime}} B^{1-\delta^{\prime \prime}}$. Since $(\alpha T,(1-\alpha) T) \times M \subset \Omega_{(1-\alpha) T} \cup(\alpha T,(1-\alpha) T) \times K$, adding the square of this estimate to the square of (13) yields def.5 with $\delta=\max \left\{\delta^{\prime}, \delta^{\prime \prime}\right\}$ and $C$ large enough.

REMARK 2.4. Even when $K \cap \bar{\Omega} \cap \partial M \neq \emptyset$, the statement of def.5 avoids the corner $\{0\} \times \partial M$, hence it should still be true and tractable. When $K \subset M$, choosing a $V$ containing $K$ avoids using (13) and yields an estimate of $\psi$ on $M_{(1-\alpha) T}$ rather the smaller set $(\alpha T,(1-\alpha) T) \times M$ in def.5.

\section{From interpolation to observability: $i i) \Rightarrow i i i)$}

Let $\mu>0, \varphi \in C_{\mathrm{comp}}^{\infty}(M)$ and $v=\mathbf{1}_{\sqrt{-\Delta} \leqslant \mu} \varphi$. Since $C_{\mathrm{comp}}^{\infty}(M)$ is dense in $L^{2}(M)$, it is enough to prove (4) for such $v$.

Let $d E_{\lambda}$ denote the projection valued measure associated to the self-adjoint operator $\sqrt{-\Delta}$. The cardinal hyperbolic sine function is the smooth function 
shc $\in C^{\infty}(\mathbb{R})$ defined by: $\operatorname{shc}(0)=0$ and $\operatorname{shc}(t)=(\exp (t)-\exp (-t)) /(2 t)$ for $t \neq 0$.

Let $F_{\mu}(t, \lambda)=t \operatorname{shc}(t \lambda) \mathbf{1}_{0 \leqslant \lambda \leqslant \mu}$. For all $j$ and $k$ in $\mathbb{N}, \lambda^{j} \partial_{t}^{k} F_{\mu} \in C\left(\mathbb{R}_{t} ; L^{\infty}\left(\mathbb{R}_{\lambda}\right)\right)$. Therefore the function $\psi$ defined by:

$$
\psi(t, x)=F_{\mu}(t, \sqrt{-\Delta}) \varphi=\sum_{k \in \mathbb{N}} \frac{t^{2 k+1}}{(2 k+1) !}(-\Delta)^{k} v,
$$

satisfies $\psi \in H^{j}\left(X_{T}\right)$ for all $j \in \mathbb{N}$ and $T>0$. Moreover, since $\varphi \in D\left((\sqrt{-\Delta})^{j}\right)$ for all $j \in \mathbb{N}$, we have $\psi(t, \cdot) \in \cap_{j \in \mathbb{N}} D\left((\sqrt{-\Delta})^{j}\right) \subset H_{0}^{1}(M)$ for all $t$.

We assume that $P=\partial_{t}^{2}+\Delta$ satisfies the interpolation inequalities in time $T$ from $\Omega$ for some $\alpha \in(0,1 / 2), \delta \in(0,1), C>0$. Since $F_{\mu}(0, \lambda)=0$ and $\psi(t, \cdot) \subset$ $H_{0}^{1}(M)$, we have $\psi=0$ on $\Gamma$, so that (5) applies to $\psi$. We have $P \psi=0$ since $\partial_{t}^{2} F_{\mu}(t, \lambda)=(\lambda)^{2} F_{\mu}(t, \lambda)$, and $\partial_{t} \psi_{\rceil t=0}=v$ since $(t \operatorname{shc}(t))_{\rceil t=0}^{\prime}=\operatorname{shc}(0)=1$, so that (5) writes:

$$
\|\psi\|_{H^{1}((\alpha T, \beta T) \times M)} \leqslant C\|\psi\|_{H^{1}\left(M_{T}\right)}^{\delta}\|v\|_{L^{2}(\Omega)}^{1-\delta}, \text { with } \beta=1-\alpha .
$$

The left-hand-side of (14) squared is greater or equal to:

$$
\begin{aligned}
\|\psi\|_{L^{2}((\alpha T, \beta T) \times M)}^{2} & =\int_{\alpha T}^{\beta T} \int\left|F_{\mu}(t, \lambda)\right|^{2} d\left(E_{\lambda} \varphi, \varphi\right) d t \\
& =\int_{\alpha T}^{\beta T} \int_{0}^{\mu}|t \operatorname{shc}(t \lambda)|^{2} d\left(E_{\lambda} \varphi, \varphi\right) d t \\
& \geqslant(\alpha T)^{2} \int_{\alpha T}^{\beta T} d t \int_{0}^{\mu} d\left(E_{\lambda} \varphi, \varphi\right)=(1-2 \alpha) \alpha^{2} T^{3}\|v\|_{L^{2}(M)}^{2} .
\end{aligned}
$$

Similarly,

$\|\psi\|_{H^{1}\left(M_{T}\right)}^{2}=\int_{0}^{T} \int_{0}^{\mu}\left(1+\lambda^{2}\right)|t \operatorname{shc}(t \lambda)|^{2} d\left(E_{\lambda} \varphi, \varphi\right) d t \leqslant T\left(1+\mu^{2}\right) e^{2 T \mu}\|v\|_{L^{2}(M)}^{2}$.

Plugging these last two estimates in (14) yields,

$$
\|v\|_{L^{2}(M)}^{2} \leqslant \frac{1+\mu^{2}}{(1-2 \alpha) \alpha^{2} T^{2}} e^{2 T \mu}\|v\|_{L^{2}(M)}^{2 \delta}\|v\|_{L^{2}(\Omega)}^{2(1-\delta)} .
$$

Therefore, for any $C_{1}>T /(1-\delta)$, there is a $C_{2}>0$ such that (4) holds.

\section{From the Laplace to the heat equation: $i i i) \Rightarrow i v$ )}

Assume that observability at low-frequencies for the Laplacian on $M$ from $\Omega$ holds. Let $\tau \in(0,1], \mu \geqslant 1$ and $u_{0} \in \mathbf{1}_{\sqrt{-\Delta} \leqslant \mu} L^{2}(M)$. For all $t \in[0, \tau]$, we may apply (4) to $v=e^{t \Delta} u_{0}$ since it is in $\mathbf{1}_{\sqrt{-\Delta} \leqslant \mu} L^{2}(M)$ :

$$
C_{2}^{2} e^{2 C_{1} \mu}\left\|e^{t \Delta} u_{0}\right\|_{L^{2}(\Omega)}^{2} \geqslant\left\|e^{t \Delta} u_{0}\right\|_{L^{2}(M)}^{2}=\int_{0}^{\mu} e^{-2 t \lambda^{2}} d\left(E_{\lambda} u_{0}, u_{0}\right) .
$$


First integrating on $[0, \tau]$ with the new variable $s=t / \tau$, then using $\tau \leqslant 1$ and finally $\int_{0}^{1} \exp (-\alpha t) d t=(1-\exp (-\alpha)) / \alpha \geqslant(2 \alpha)^{-1}$ for $\alpha \geqslant \ln 2$ yields:

$$
\begin{aligned}
C_{2}^{2} e^{2 C_{1} \mu}\left\|e^{t \Delta} u_{0}\right\|_{L^{2}\left(\Omega_{\tau}\right)}^{2} & \geqslant \tau \int_{0}^{1} \int_{0}^{\mu} e^{-2 \tau s \lambda^{2}} d\left(E_{\lambda} u_{0}, u_{0}\right) d s \\
& \geqslant \tau \int_{0}^{1} e^{-2 s \mu^{2}} d s \int_{0}^{\mu} d\left(E_{\lambda} u_{0}, u_{0}\right) \geqslant \frac{\tau}{4 \mu^{2}}\left\|u_{0}\right\|_{L^{2}(M)}^{2} .
\end{aligned}
$$

Therefore, for any $D_{1}>C_{1}$, there is a $D_{2}>0$ such that (3) holds.

\section{From low-frequencies to full observability: $i v) \Rightarrow v$ )}

By duality (cf. [DR77]), def.3 is equivalent to the following null-controllability at low frequencies (cf. def.1): for all $\tau \in(0,1]$ and $\mu \geqslant 1$, there is a bounded operator $S_{\mu}^{\tau}: L^{2}(M) \rightarrow L^{2}(\mathbb{R} \times M)$ such that for all $\phi_{0} \in 1_{\sqrt{-\Delta} \leqslant \mu} L^{2}(M)$, the solution $\phi \in C^{0}\left([0, \infty), L^{2}(M)\right)$ of (1) with control function $f=S_{\mu}^{\tau} \phi_{0}$ satisfies $\mathbf{1}_{\sqrt{-\Delta} \leqslant \mu} \phi=0$ at $t=\tau$. Moreover, we have the cost estimate: $\left\|S_{\mu}^{\tau}\right\| \leqslant \frac{D_{2}}{\sqrt{\tau}} e^{D_{1} \mu}$.

We introduce a dyadic scale of frequencies $\mu_{k}=2^{k}(k \in \mathbb{N})$ and a sequence of time intervals $\tau_{k}=\sigma_{\delta} T / \mu_{k}^{\delta}$ where $\delta \in(0,1)$ and $\sigma_{\delta}=\left(2 \sum_{k \in \mathbb{N}} 2^{-k \delta}\right)^{-1}>0$, so that the sequence of times defined recursively by $T_{0}=0$ and $T_{k+1}=T_{k}+$ $2 \tau_{k}$ converges to $T$. The strategy of Lebeau and Robbiano in [LR95] is to steer the initial state $\phi_{0}$ to 0 through the sequence of states $\phi_{k}=\phi\left(T_{k}\right) \in$ $\mathbf{1}_{\sqrt{-\Delta}>\mu_{k-1}} L^{2}(M)$ at frequencies converging to infinity by applying recursively the control function $f_{k}=S_{\mu_{k}}^{\tau_{k}} \phi_{k}$ to $\phi_{k}$ during a time $\tau_{k}$ and zero control during a time $\tau_{k}$. This strategy is succesful if $\phi_{k}$ tends to zero and the control function $f(t)=\sum_{k} \mathbf{1}_{0 \leqslant t-T_{k} \leqslant \tau_{k}} f_{k}(t)$ is in $L^{2}\left(0, T ; L^{2}(M)\right)$. Since the cost estimate above implies $\left\|S_{\mu_{k}}^{\tau_{k}}\right\| \leqslant D_{2} e^{D_{1} \mu_{k}} / \sqrt{\tau_{k}}$, it only remains to check that:

$$
\varepsilon_{k}=\left\|\phi_{k}\right\| \text { and } C_{k}=D e^{D_{1} \mu_{k}} / \mu_{k}^{\delta} \text { satisfy } \lim _{k} \varepsilon_{k}=0 \text { and } \sum_{k \in \mathbb{N}} C_{k}^{2} \varepsilon_{k}^{2}<\infty .
$$

Since $\mathbf{1}_{\sqrt{-\Delta} \leqslant \mu_{k}} \phi\left(T_{k}+\tau_{k}\right)=0$, we have $\varepsilon_{k+1} \leqslant e^{-\tau_{k} \mu_{k}^{2}}\left\|\phi\left(T_{k}+\tau_{k}\right)\right\|$. The expression of $\phi\left(T_{k}+\tau_{k}\right)$ in terms of the source term $f_{k}$ (Duhamel's formula) and $\left\|e^{t \Delta}\right\| \leqslant$ 1 (contractivity of the heat semigroup) imply $\left\|\phi\left(T_{k}+\tau_{k}\right)\right\| \leqslant 2\left(\varepsilon_{k}+\sqrt{\tau_{k}}\left\|f_{k}\right\|\right)$. Therefore $\varepsilon_{k+1} \leqslant 2 e^{-\tau_{k} \mu_{k}^{2}}\left(1+\sqrt{\tau_{k}} C_{k}\right) \varepsilon_{k}$. Since $C_{k+1} / C_{k}=e^{D_{1} \mu_{k}} / 2^{\delta / 2}$, we deduce:

$$
\frac{C_{k+1} \varepsilon_{k+1}}{C_{k} \varepsilon_{k}} \leqslant 2 e^{-\tau_{k} \mu_{k}^{2}}\left(1+D_{2} e^{D_{1} \mu_{k}}\right) e^{D_{1} \mu_{k}} / 2^{\delta / 2} \leqslant D \exp \left(2 D_{1} \mu_{k}-\sigma_{\delta} T \mu_{k}^{2-\delta}\right),
$$

for some $D>0$. Since $2-\delta>1$ this implies $\sum_{k \in \mathbb{N}} C_{k}^{2} \varepsilon_{k}^{2}<\infty$, which proves (15) and completes the proof of the null-controllability in def.1.

\section{Necessary condition: $v) \Rightarrow v i)$}

The main ingredient is an $L^{2}$ Gaussian upper bound on the heat kernel. Following [CGT82] (and section 6.2 in the book [Tay96]), the finite propagation 
speed of solutions to the wave equation yields:

$$
\left\|\mathbf{1}_{U} e^{t \Delta} \mathbf{1}_{V}\right\|_{\mathcal{L}\left(L^{2}(M)\right)} \leqslant \frac{1}{\sqrt{4 \pi t}} \int_{|s| \geqslant \rho} \exp \left(-\frac{s^{2}}{4 t}\right) d s \leqslant \exp \left(-\frac{\rho^{2}}{4 t}\right),
$$

for any open sets $U$ and $V$ in $M$ such that $\rho=\operatorname{dist}(U, V)=\inf _{(x, y) \in U \times V} \operatorname{dist}(x, y)$.

Assume that the sequence $\left(B_{k}\right)_{k \in \mathbb{N}}$ of open subsets of $\bar{M}$ goes away from $\Omega$ without shrinking with a factor $T$ (def.6). For any $k \in \mathbb{N}$, let $\tilde{\Delta}_{k}$ denote the Dirichlet Laplacian on $B_{k}$ with domain $D\left(\tilde{\Delta}_{k}\right)=H_{0}^{1}\left(B_{k}\right) \cap H^{2}\left(B_{k}\right)$ and let $\Delta_{k}$ denote its extension to $L^{2}(M)$ by zero. Let $\tilde{u}_{k} \in L^{2}\left(B_{k}\right)$ be an eigenfunction of $-\tilde{\Delta}_{k}$ with unit norm associated to its first eigenvalue $\lambda^{*}\left(B_{k}\right)>0$, and let $u_{k} \in L^{2}(M)$ be the extension of $\tilde{u}_{k}$ by zero. Since $\Delta_{k}$ is an extension of $\Delta$, we have:

$$
\left\|e^{T \Delta} u_{k}\right\|_{L^{2}(M)} \geqslant\left\|e^{T \Delta_{k}} u_{k}\right\|_{L^{2}(M)}=\left\|e^{T \tilde{\Delta}_{k}} \tilde{u}_{k}\right\|_{L^{2}\left(B_{k}\right)}=\exp \left(-\lambda^{*}\left(B_{k}\right) T\right) .
$$

Partitioning $\Omega$ into slices $U=\left\{x \in \Omega \mid \rho \leqslant \operatorname{dist}\left(x, B_{k}\right)<\rho+\varepsilon\right\}$ of thickness $\varepsilon>$ 0 , applying (16) to each $U$ with $V=B_{k}$, summing up and letting $\varepsilon \rightarrow 0$ yields:

$$
\left\|e^{t \Delta} u_{k}\right\|_{L^{2}\left(\Omega_{T}\right)} \leqslant \sqrt{T} \int_{\Omega \backslash B} \exp \left(-\frac{\operatorname{dist}(x, B)^{2}}{2 T}\right) d x=\sqrt{T} \exp \left(-\frac{\overline{\operatorname{dist}}_{T}\left(B_{k}, \Omega\right)^{2}}{4 T}\right) .
$$

Plugging the last two inequalities in (2) contradicts the limit in def.6. Therefore the heat equation on $M$ is not final-observable in time $T$ from $\Omega$.

\section{Finer sufficient condition: $\left.\left.i^{\prime}\right) \Rightarrow i i\right)$}

In this section, we prove that th. 2 results from the following covering lemma obtained by combining the argument of the proof of lem.1.2 in [Shu92] with Gromov's volume comparision theorem (prop.4.1.iii in [CGT82] or th.3.10 in [Cha93]):

Lemma 7.1. If the Ricci curvature of $M$ is bounded below there are $\rho_{0}>0$ and $N \in \mathbb{N}^{*}$ such that for any $\rho \in\left(0, \rho_{0}\right)$ there is a sequence of balls $B_{\rho}\left(y_{j}\right)$ $(j \in \mathbb{N})$ with center $y_{j} \in \bar{M}$ and radius $\rho$ covering $\bar{M}$ such that each ball $B_{\rho}\left(y_{j}\right)$ intersects no more than $N$ other balls $B_{\rho}\left(y_{k}\right)(k \neq i)$.

Let $B_{\rho}\left(y_{j}\right)(j \in \mathbb{N})$ be a covering with multiplicity not greater than $N$ as in this lemma. Assuming i'), for each $j \in \mathbb{N}$, there is an $x_{j} \in \Omega$ such that $B_{r}\left(x_{j}\right) \in$ $\Omega$ and $\operatorname{dist}\left(y_{j}, B_{r}\left(x_{j}\right)\right) \leqslant d$. Setting $R=r+d+\rho$ implies $B_{\rho}\left(y_{j}\right) \subset B_{R}\left(x_{j}\right)$, so that, fixing $\alpha \in(0,1 / 2)$, applying (6) with $x=x_{j}$ yields $\forall j, \forall \psi \in H^{2}\left(M_{T}\right)$ s.t. $\psi=0$ on $\Gamma$,

$$
\|\psi\|_{H^{1}\left((\alpha T,(1-\alpha) T) \times B_{\rho}\left(y_{j}\right)\right)} \leqslant C\|\psi\|_{H^{1}\left(M_{T}\right)}^{\delta_{1}}\left(\|P \psi\|_{L^{2}\left(M_{T}\right)}+\left\|\partial_{t} \psi_{\rceil t=0}\right\|_{L^{2}(\Omega)}\right)^{1-\delta} .
$$

Summing up over $j$ implies:

$$
\|\psi\|_{H^{1}((\alpha T,(1-\alpha) T) \times \bar{M})} \leqslant N C\|\psi\|_{H^{1}\left(M_{T}\right)}^{\delta}\left(\|P \psi\|_{L^{2}\left(M_{T}\right)}+\left\|\partial_{t} \psi_{\urcorner t=0}\right\|_{L^{2}(\Omega)}\right)^{1-\delta} .
$$

Therefore def.5 holds and the proof of $\left.i^{\prime}\right) \Rightarrow i i$ ) is completed. 


\section{References}

[CDMZ01] V. R. Cabanillas, S. B. De Menezes, and E. Zuazua, Null controllability in unbounded domains for the semilinear heat equation with nonlinearities involving gradient terms, J. Optim. Theory Appl. 110 (2001), 245-264.

[CGT82] J. Cheeger, M. Gromov, and M. Taylor, Finite propagation speed, kernel estimates for functions of the Laplace operator, and the geometry of complete Riemannian manifolds, J. Differential Geom. 17 (1982), 15-53.

[Cha93] I. Chavel, Riemannian geometry: a modern introduction, Cambridge Tracts in Mathematics, 108, Cambridge U.P., Cambridge, 1993.

[Che75] S. Y. Cheng, Eigenvalue comparision theorems and its geometric applications, Math. Zeit. 143 (1975), 289-297.

[DR77] S. Dolecki and D. L. Russell, A general theory of observation and control, SIAM J. Control Optimization 15 (1977), 185-220.

[JL99] D. Jerison and G. Lebeau, Nodal sets of sums of eigenfunctions, Harmonic analysis and partial differential equations (Chicago, IL, 1996), Univ. Chicago Press, Chicago, IL, 1999, 223-239.

[Lin90] F.-H. Lin, A uniqueness theorem for parabolic equations, Comm. Pure Appl. Math. 43 (1990), 127-136.

[Lin91] Nodal sets for solutions of elliptic and parabolic equations, Comm. Pure Appl. Math. 44 (1991), 287-308.

[LR95] G. Lebeau and L. Robbiano, Contrôle exact de l'équation de la chaleur, Comm. Partial Differential Equations 20 (1995), 335-356.

[LZ98] G. Lebeau and E. Zuazua, Null-controllability of a system of linear thermoelasticity, Arch. Rational Mech. Anal. 141 (1998), 297-329.

[Mil04a] L. Miller, How violent are fast controls for Schrödinger and plates vibrations ?, Arch. Ration. Mech. Anal. 172 (2004), 429-456.

[Mil04b] On the controllability of the heat equation in unbounded domains, to appear in Bull. Sci. Math., http://hal.ccsd.cnrs.fr/ccsd-00001475, preprint, 2004.

[MZ03] S. Micu and E. Zuazua, Null-controllability of the heat equation in unbounded domains, Unsolved Problems in Mathematical Systems and Control Theory (V.D. Blondel and A. Megretski, eds.), Princeton U.P., 2003, to appear 2004.

[Rob95] L. Robbiano, Fonction de coût et contrôle des solutions des équations hyperboliques, Asymptotic Anal. 10 (1995), 95-115.

[Shu92] M. A. Shubin, Spectral theory of elliptic operators on noncompact manifolds, Astérisque 5 (1992), 35-108, Méthodes semi-classiques, Vol. 1 (Nantes, 1991).

[Tay96] M. E. Taylor, Partial differential equations. I, Applied Mathematical Sciences, 115, Springer-Verlag, New York, 1996, Basic theory.

Équipe Modal'X, EA 3454, Université Paris X, Bât. G, 200 Av. de la République, 92001 Nanterre, France.

Centre de Mathématiques Laurent Schwartz, UMR CNRS 7640, École PolytechNIQUe, 91128 Palaiseau, France.

E-mail address: miller@math.polytechnique.fr 